\title{
Translocator Protein (18 kDa): A Promising Therapeutic Target and Diagnostic Tool for Cardiovascular Diseases
}

\author{
Xiaolong Qi, ${ }^{1,2}$ Jiahong Xu, ${ }^{3}$ Fei Wang, ${ }^{2}$ and Junjie Xiao ${ }^{1}$ \\ ${ }^{1}$ Cell Metabolism Lab, School of Life Science, Shanghai University, 333 Nan Chen Road, Shanghai 200444, China \\ ${ }^{2}$ Department of Gastroenterology, Tongji hospital, Tongji University School of Medicine, Shanghai 200065, China \\ ${ }^{3}$ Department of Cardiology, Tongji Hospital, Tongji University School of Medicine, Shanghai 200065, China
}

Correspondence should be addressed to Junjie Xiao, junjiexiao@shu.edu.cn

Received 28 June 2012; Revised 22 October 2012; Accepted 4 November 2012

Academic Editor: Jose Magalhaes

Copyright (C) 2012 Xiaolong Qi et al. This is an open access article distributed under the Creative Commons Attribution License, which permits unrestricted use, distribution, and reproduction in any medium, provided the original work is properly cited.

\begin{abstract}
The translocator protein $(18 \mathrm{kDa})$ (TSPO) is a five transmembrane domain protein in mitochondria, abundantly expressed in a variety of organs and tissues. TSPO contributes to a wide range of biological processes, including cholesterol transportation, mitochondrial membrane potential and respiratory chain regulation, apoptosis, and oxidative stress. Recent studies have demonstrated that TSPO might also be involved in the physiological regulation of cardiac chronotropy and inotropy. Accordingly, TSPO ligands play significant roles in protecting the cardiovascular systems under pathological conditions through cardiac electrical activity retention, intracellular calcium maintenance, mitochondrial energy provision, mitochondrial membrane potential equilibrium, and reactive oxygen species inhibition. This paper focuses on the physiological and pathological characteristics of TSPO in the cardiovascular systems and also summarizes the properties of TSPO ligands. TSPO represents a potential therapeutic target and diagnostic tool for cardiovascular diseases including arrhythmia, myocardial infarction, cardiac hypertrophy, atherosclerosis, myocarditis, and large vessel vasculitis.
\end{abstract}

\section{Introduction}

The translocator protein $(18 \mathrm{kDa})(\mathrm{TSPO})$ is a five transmembrane domain protein in mitochondria, which was previously known as peripheral benzodiazepine receptor (PBR) [1-3]. TSPO exists in a variety of species and is abundantly expressed in human organs [4-6]. It is involved in a wide range of biological processes, including controlling the synthesis of steroids, regulating mitochondrial membrane potential and respiratory chain, modulating voltagedependent calcium channels, controlling immune response, apoptosis, and oxidative stress [1, 3, 4]. A large number of studies have demonstrated that TSPO was involved in regulating cardiac chronotropy and inotropy [4, 7-15].

Cardiovascular diseases (CVDs) are a global health issue causing tremendous economic burdens [16-18]. Considering the high abundance and significant physiological roles in the heart, TSPO has been recognized as a promising therapeutic target and diagnostic tool for CVD [7-15].
Emerging lines of evidence have suggested that TSPO plays significant roles in CVD including arrhythmia, myocardial infarction (MI), cardiac hypertrophy $(\mathrm{CH})$, atherosclerosis, myocarditis, and large vessel vasculitis (LVV) [7-15]. The mechanisms responsible for its cardioprotective effects include cardiac electrical activity retention, intracellular calcium maintenance, mitochondrial energy provision, mitochondrial membrane potential equilibrium, and reactive oxygen species inhibition $[1,3,4]$. Here, we reviewed the physiological and pathological characteristics of TSPO and also summarized its role in CVD, hoping to offer a foundation for further studies on the development of TSPO as a therapeutic target and diagnostic tool for CVD.

\section{TSPO}

2.1. Rename. TSPO is a new name of PBR, which addressed the shortcomings and misrepresentations of PBR in the 
scientific community mainly for historical reasons. Although previously this protein had multiple names known as mitochondrial diazepam-binding inhibitor (DBI) receptor complex, PK11195-binding sites, isoquinoline-binding protein (IBP), pk18, and $\omega 3$ receptor and so forth $[3,4,7]$, none reflects its true nature and function. Among three main structure-function relationships for the PBR: (i) cholesterol binding and transport; (ii) protein import; and (iii) porphyrin binding and transport, transporting molecules from the outside to the inside are its major function $[1,3,4]$. The new nomenclature represents more accurately its subcellular roles and putative molecular functions. Thus, a consensus on the new name of PBR known as TSPO was reached in 2006 by the HUGO Gene Nomenclature Committee [3], which referred only to the $18 \mathrm{kDa}$ protein and the minimal functional unit (binding site) of all known PBR ligands, regardless of its functional associations with other proteins [1-5].

2.2. Structure. TSPO, which consists of 169 amino acids and five transmembrane domain, can form a complex with voltage-dependent anion channel (VDAC, $32 \mathrm{kDa}$ ) at the outer membrane and adenine nucleotide translocator (ANT, $30 \mathrm{kDa}$ ) at the inner membrane of the mitochondria (Figure 1) $[2,4]$. Structurally, the complex is also a combination of creatine kinase, proteins of the Bcl-2 family, PBRassociated protein 1 and protein 7. TSPO, VDAC, and ANT show a high degree of homology between various species [1]. Intracellularly, TSPO locates primarily on mitochondrial membranes, especially at the connection sites between the outer and inner membranes. Traditionally, VDAC and ANT are considered as the core components of the mitochondrial permeability transition pore (MPTP) [1]. However, a recent study exploring the effects of mammalian VDAC deletion on mitochondrial-dependent cell death proved that the wildtype and VDAC-deficient mitochondria and cells exhibited equivalent cytochrome $c$ release, caspase cleavage, $\mathrm{Ca}^{2+}$, and oxidative stress-induced mitochondrial permeability transition [19]. In addition, another study also showed that mitochondria lacking ANT could still be induced to undergo permeability transition. Moreover, hepatocytes without ANT remained competent to respond to various initiators of cell death [20]. Therefore, VDAC and ANT appear to be regulators rather than indispensable constituents of MPTP. Interestingly, several studies have demonstrated that TSPO may modulate the function of VDAC and ANT $[1,21,22]$.

2.3. Distribution. TSPO is the product of family genes that is evolutionarily conserved from bacteria to human and exists across various species including insects, mollusks, fishes, amphibians, birds, and mammals $[1,4,5,23,24]$. TSPO abundantly expresses in adrenal, kidney, brain, and heart [1]. In the cardiovascular lumen, it is mainly present in platelets, erythrocytes, lymphocytes, and monocytes $[1,4]$. TSPO can also be found in the walls of the cardiovascular system, such as endothelium, striated muscle, and smooth muscle [1$4,6]$.
Mitochondrion is the primarily subcellular location of TSPO. However, several studies have demonstrated that TSPO could also be found in the nuclear fractions and plasma membrane [1-4]. Hence, the widespread expression of TSPO in organ, cellular, and subcellular level suggests its essential functions in biological process $[1,3,4]$.

2.4. Functions. A wide spectrum of putative functions of TSPO have been suggested after binding to high-affinity ligands and cholesterol (Figure 1), such as cholesterol transportation, steroidogenesis regulation, porphyrin transportation, heme synthesis, anion transportation, cell growth and differentiation, cancer cell proliferation, apoptosis, mitochondrial membrane potential and respiratory chain regulation, voltage-dependent calcium channels modulation, and microglial activation related to brain damage and immune response $[1-4,25,26]$. However, the exact pathways regarding how TSPO is involved in those functions are still unclear $[1-5,27,28]$.

Recently, a growing body of evidence indicates that TSPO ligands may play vital protective roles in cardiovascular systems through cardiac electrical activity retention, intracellular calcium maintenance, mitochondrial energy provision, mitochondrial membrane potential $\left(\Delta \Psi_{\mathrm{m}}\right)$ equilibrium, and reactive oxygen species (ROS) release inhibition $[1,4,7-$ 11]. Due to the significant clinical application potential of TSPO ligands, they represent a novel potential treatment for preventing pathological dysfunctions of CVD.

2.5. Physiological Roles in the Heart. TSPO has vital physiological roles in the heart. Accumulating lines of evidence have demonstrated that TSPO ligands, such as 1-(2chlorophenyl)-Nmethyl- N-(1-methyl-propyl)-3- isoquinoline carboxamide (PK 11195) and $4^{\prime}$-chlorodiazepam (4CIDzp), could regulate cardiac chronotropy (heart rate) and inotropy (contractile force) [29, 30]. In addition, a proposed mechanism of alteration in cardiac action potential duration and contractility has been investigated as a result of the interaction between TSPO and voltage-gated $\mathrm{Ca}^{2+}$ channels. TSPO is a potential pharmacologic receptor and its ligands may be essential in calcium transportation [31].

TSPO ligands, such as PK11195 which may cause adverse effects in the heart, have been identified as agonists, while ligands like 4-ClDzp that has cardiac protective effects have been known as antagonist $[8,32]$. Although TSPO ligands at low concentrations seem to have no influence in heart rate, the negative chronotropy can be found at high concentrations [30, 33, 34]. PK11195 alone does not alter either inotropic effect or coronary flow velocity, while the negative inotropic effects induced by 4-ClDzp could be antagonized by PK11195 in various models including papillary muscle of guinea pigs, isolated perfused rat and rabbit hearts, and isolated canine right atrium [30, 31, 3538 .

In summary, TSPO plays significant roles in the physiological regulation of the heart. Interestingly, the negative chronotropic effects of TSPO ligand such as 4-ClDzp that have been proved in the canine [29, 39] and rabbit [37] 


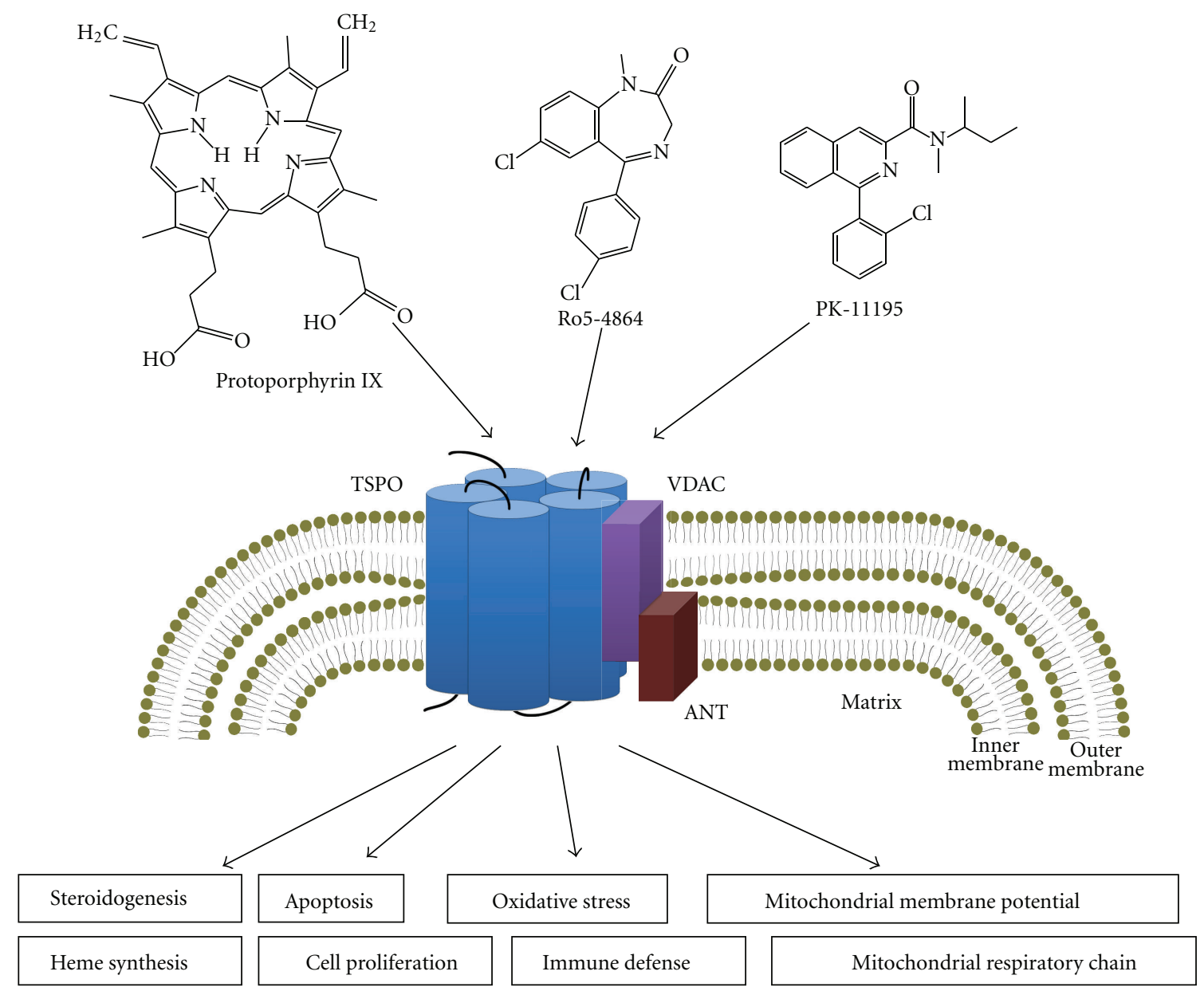

FIGURE 1: Structure and potential functions of TSPO.

cannot be identified in rat [30, 33] and guinea pig [34]. Therefore, the exact physiological role of TSPO in the heart is still unclear as great difference among species might exist.

2.6. Ligands Classification. TSPO ligands have been widely used to investigate the role of TSPO in cardiovascular diseases $[1,4,40]$. Supposed endogenous ligands of TSPO include protoporphyrin IX, diazepam binding inhibitor (DBI), triakontatetraneuropeptide (TTN), and phospholipase A2 (PLA2) (Figure 2(a)). Classical synthetic ligands for TSPO include 7-chloro-5-(4-chlorophenyl)-1,3dihydro-1-methyl-2H-1,4-benzodiazepin-2-one (Ro54864) or 4-ClDzp and PK 11195 (Figure 2(b)). Novel TSPO ligands in addition to the classical synthetic ligands have also been developed, such as N,N-di-n-hexyl 2-(4-fluorophenyl) indole-3-acetamide (FGIN-1-27) and 7-chloro-N,N, 5trimethyl-4-oxo-3-phenyl-3,5-dihydro-4H-pyridazino[4,5b] indole-1-acetamide (SSR180575) (Figure 2(c)). Although FGIN-1-27 and SSR180575 both possess steroidogenic properties, the former is expressed as proapoptotic ligand while the latter shows antiapoptotic property $[26,41]$. Recently, 3,5-Seco-4-nor-cholestan-5-one oxime-3-ol (TRO40303) (Figure 2(c)), which is identified initially for neuroprotective properties, has been found to be a novel TSPO ligand binding specifically to the cholesterol site and exhibits the cardioprotective properties [42].

\section{TSPO as a Therapeutic Target and Diagnostic Tool for CVD}

3.1. Arrhythmia. Arrhythmia refers to the abnormal electrical impulses which may happen too fast, too slowly, or erratically, among which ventricular fibrillation (VF) and atrial fibrillation (AF) are most commonly reported arrhythmias associated with TSPO [43-45]. Over the last two decades, a growing body of evidence suggests that cardiac mitochondria dysfunction is a significant cause for arrhythmias $[7,8,32,46]$. TSPO, which spans the inner and outer mitochondrial membranes, is one of the most significant targets involved in the regulation mitochondrial functions, including mitochondrial respiratory chain regulation, ROS generation and release, and inner membrane anion channels (IMACs) regulation (Figure 3) [4, 47-49].

IMAC can induce the opening of other inner membrane channels during oxidative stress and may be an effective target to prevent the metabolic oscillations [9]. Although 


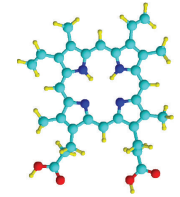

Protoporphyrin IX

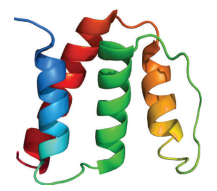

DBI

(a)

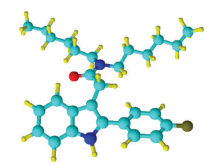

FGIN-1-27

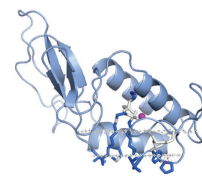

PLA2

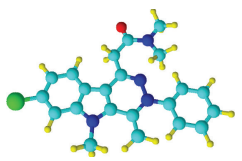

SSR180575

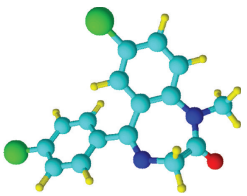

Ro5-4864

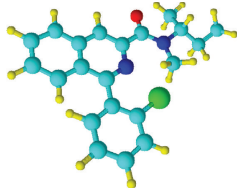

PK-11195

(b)

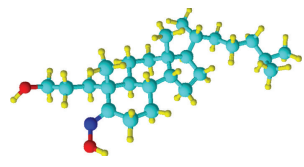

TRO40303

(c)

FIgURE 2: Three-dimensional structures of representative TSPO ligands. (a) endogenous ligands, such as protoporphyrin IX, diazepam binding inhibitor (DBI), and phospholipase A2 (PLA2); (b) classical synthetic ligands, such as 7-chloro-5-(4-chlorophenyl)-1,3-dihydro1-methyl-2H-1,4-benzodiazepin-2-one (Ro5-4864) and PK 11195; (c) novel ligands, such as N,N-di-n-hexyl 2-(4-fluorophenyl)indole3-acetamide (FGIN-1-27), 7-chloro-N,N,5-trimethyl-4-oxo-3-phenyl-3,5-dihydro-4H-pyridazino[4,5-b]-indole-1-acetamide (SSR180575) and 3,5-Seco-4-nor-cholestan-5-one oxime-3-ol (TRO40303).

the accurate structure of IMAC is not clear, the sensitivity of the anion channel regulated by TSPO ligands suggests that IMAC subunits are associated with TSPO $[9,10]$. In addition, a local burst of mitochondrial ROS during ischemia/reperfusion (I/R) can lead to the increases of ROS production and oscillations in $\Delta \Psi_{\mathrm{m}}$ (Figure 3), which could be blocked by TSPO ligands that have been previously known to block the activity of IMAC, such as PK11195 and 4-ClDzp [50-54]. Other investigations using different species in reperfusion-induced VF models have demonstrated that the progressive action potential shortening followed by membrane inexcitablity in ischemia and VF upon reperfusion could be reduced or even eliminated by 4-ClDzp treatment before reperfusion. By contrast, FGIN-1-27 promotes $\Delta \Psi_{\mathrm{m}}$ depolarization, exacerbates I/R-induced electrophysiological changes and promotes VF $[8,37,55]$. Therefore, TSPO may be an effective therapeutic target for VF.

As for AF, our group has demonstrated that inhibition of TSPO by its antagonist could significantly reduce the incidence of AF induced by ischemia-, stretch-, and cholinergic agitation [7]. In addition, the suppression of TSPO in atrial muscle cell lines could ameliorate the cytoplasm $\mathrm{Ca}^{2+}$ overload and energy compromise facing to chemical ischemia or cholinergic agitation [7]. Therefore, TSPO antagonists may be a novel treatment for various AF in the near future.

In summary, blocking mitochondrial IMAC through TSPO antagonists can effectively prevent mitochondrial ROS-induced ROS release and the loss of $\Delta \Psi_{\mathrm{m}}$ triggered by oxidative stress $[7,8]$. This effect is correlated with preservation of the action potential during ischemia as well as restoration of normal electrical activity upon reperfusion [4, 7, 47-49]. Therefore, suppression of arrhythmias, including VF and AF, by stabilization of $\Delta \Psi_{\mathrm{m}}$ and inhibition of ROS overload with TSPO ligands offers us a novel therapy for arrhythmia.
3.2. Myocardial Infarction. Myocardial infarction (MI) is responsible for the majority of cardiovascular mortality. The irreversible cell injury including necrosis and apoptosis might be induced by reperfusion therapy, a major therapy for MI [56-58]. Thus, investigating a novel therapy for I/R will definitely benefit MI. I/R injury causes mitochondrial swelling and the release of cytochrome $c$, which is involved in the mPTP opening [59-62]. mPTP plays a significant role in the generation of necrotic and apoptotic cell death $[59,60]$. Administration of cyclosporine A, a high-affinity inhibitor of cyclophilins that desensitizes the MPTP to the inducing effects of $\mathrm{Ca}^{2+}$, can attenuate several indices of MI [60-62]. According to a study of photodynamic events mediated by porphyrins at mPTP-regulating His and Cys residues, the inactivation of His and Cys residues resulting from matrix porphyrin could be reactivated at high light dose through a different porphyrin site, which was specifically contributed by the outer mitochondrial membrane through TSPO $[60,63]$. Therefore, TSPO may play a dual role in mPTP regulation including (i) as a transport protein for PTP-active compounds that are transferred to their regulatory site in the inner mitochondrial membrane or matrix and (ii) as a mPTP regulatory protein when binding to its selective ligands like porphyrins [60]. Considering the role of TSPO in $\mathrm{mPTP}$ regulation, it holds great promise as a therapeutic target for MI.

Our previous study has provided direct evidence that 4CIDzp accelerated the recovery of left ventricular developed pressure (LVDP), left ventricular end-diastolic pressure (LVEDP), and maximal time derivatives of pressure measured during contraction, relaxation $( \pm d P / d t \max )$ in $\mathrm{I} / \mathrm{R}$ [64]. The mPTP opening is also reduced by 4 -ClDzp, and thereafter a decreased ROS level could be observed [64]. Similarly, other studies have showed that 4-ClDzp reduced infarct size in a dose-dependent manner either in global or regional models of myocardial I/R in rat [65]. Thus, 4-ClDzp 


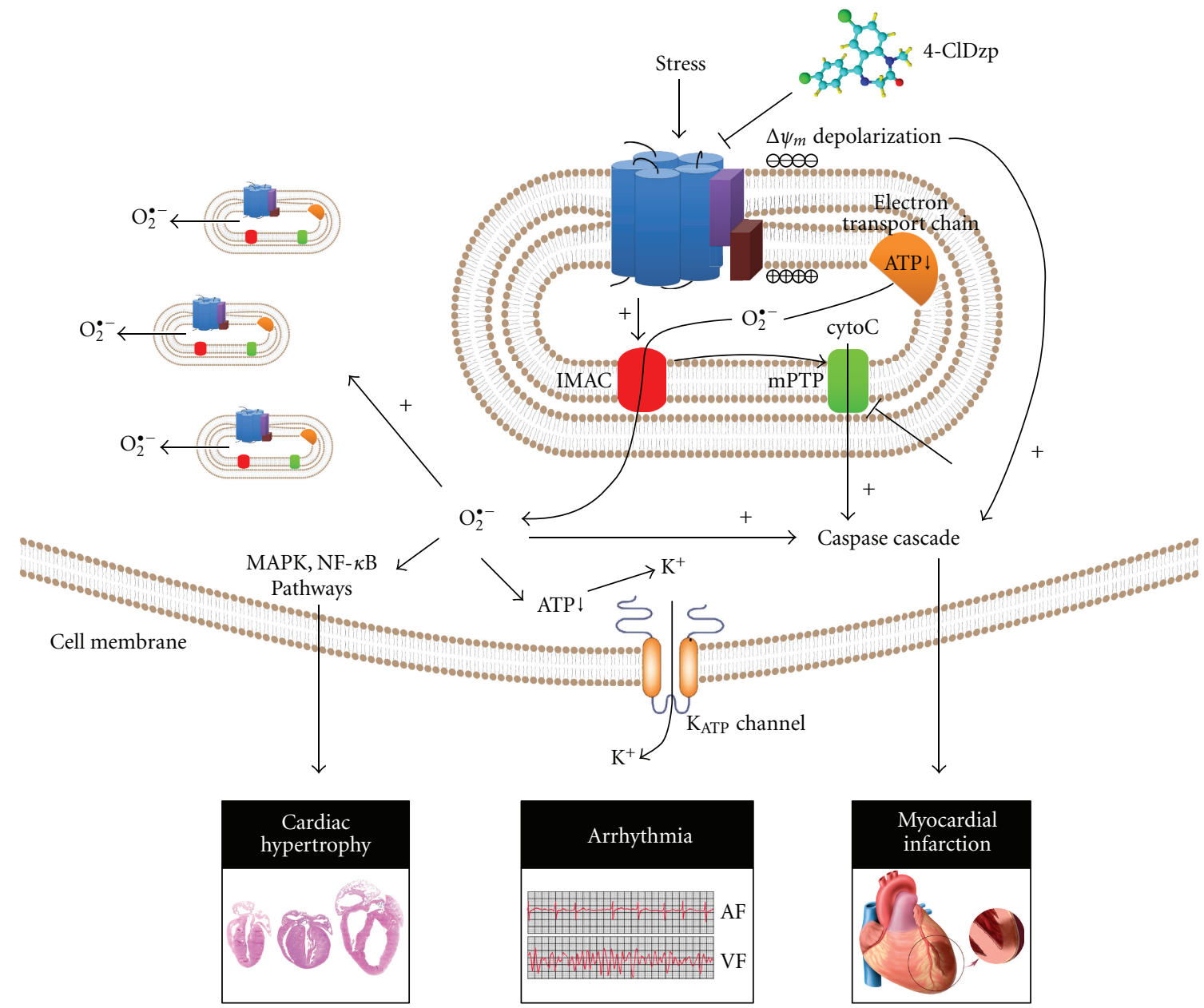

FIGURE 3: TSPO is a therapeutic target for arrhythmia, myocardial infarction, and cardiac hypertrophy. Ischemia/reperfusion rapidly induces reactive oxygen species (ROS) production from the electron transport chain, and ROS are released to mitochondrial matrix. A local burst of mitochondrial ROS leads to the increase of ROS production and oscillations in mitochondrial membrane potential $\left(\Delta \Psi_{\mathrm{m}}\right)$, which can destabilize neighboring mitochondrial membrane by inducing the release of ROS. The collapses of $\Delta \Psi_{\mathrm{m}}$ are regulated by inner membrane anion channels (IMACs) opening, which is key in the genesis of arrhythmia. The burst of ROS in cellular matrix activates a broad variety of signaling kinases and transcription factors related to cardiac hypertrophy. The imbalance of IMAC influences the opening of mitochondrial permeability transition pore ( $\mathrm{mPTP}$ ), leading to the release of cytochrome $c$ and activation of caspase cascade, and contributes to myocardial infarction. Targeting TSPO can reverse the above changes. Thus, TSPO is a therapeutic target for arrhythmia, myocardial infarction, and cardiac hypertrophy through reducing ROS.

might protect MI through reducing apoptosis, restoring mitochondrial recovery, improving oxidative phosphorylation parameters, reducing cytochrome $c$ and apoptosisinducing factor releases, and increasing the resistance of mitochondria to $\mathrm{Ca}^{2+}$-induced mPTP opening [62-65].

3.3. Cardiac Hypertrophy. Cardiac hypertrophy $(\mathrm{CH})$ is a common pathological feature of several major cardiovascular diseases and a physiological adaptation to increased hemodynamic overload [66-69]. Oxidative stress has been identified as one of the key contributing factors in myocardial structural damage and cardiac remodeling in $\mathrm{CH}$. TSPO is associated with the regulation of cellular oxidative stress [69], which can activate a broad variety of signaling kinases and transcription factors related to $\mathrm{CH}$, such as MAPK and NF$\kappa \mathrm{B}$ (Figure 3) [66]. A strong link between oxidative stress and extracellular matrix remodeling has been reported [66]. Besides, TSPO has been found to be associated with the protection of cells against oxygen radical damage and the regulation of mPTP opening $[65,66]$. 4-ClDzp has been shown to inhibit mPTP opening and prevent isoprenalineinduced $\mathrm{CH}$ as a result of the reduction of heart weight to body weight ratio, left ventricular wall thickness, and myocyte size on male Wistar rats $[65,70]$. Moreover, 4ClDzp could attenuate the increase of interstitial fibrosis, lipid peroxidations, endogenous antioxidants, and $\beta$ myosin heavy chain induced by isoprenaline [11]. Thus, TSPO is a potential target for $\mathrm{CH}$ treatment.

3.4. Atherosclerosis. Atherosclerosis is characterized with inflammatory infiltration of macrophages, dendritic cells, and activated $\mathrm{T}$ cells, which is initiated by lipids deposition 
in the subendothelial layer of the arterial wall $[71,72]$. Macrophages specifically in the atherosclerotic plaque contribute to the local inflammatory responses by secreting proinflammatory cytokines [73-75]. Activated macrophages have been demonstrated with high TSPO expression levels [76]. Several studies have investigated the uptake of $\left[{ }^{11} \mathrm{C}\right]$ PK11195, a TSPO radioligand, in atherosclerotic plaques, and predicted its potential diagnostic value in atherosclerosis. They found that the uptake rate of $\left[{ }^{11} \mathrm{C}\right] \mathrm{PK} 11195$ in inflammatory regions was much higher than other areas $[76,77]$. The high level of TSPO in plaque macrophages indicates a diagnostic tool through noninvasive PET imaging to predict the morphology and pathogenesis of pre-rupture atherosclerosis based on TSPO $[12,75]$. Recently, the combination of ${ }^{11} \mathrm{C}-\mathrm{PK} 11195$ PET with contrast-enhanced CT angiography offers a comprehensive assessment of plaque structure, composition, and biological activity [76, 77]. It allows the distinction between symptomatic vulnerable plaques and asymptomatic plaques with a high positive predictive value and risk stratification of asymptomatic carotid stenosis, ischemic cerebrovascular events with low CT attenuation, and high ${ }^{11} \mathrm{C}-\mathrm{PK} 11195$ uptake $[76,77]$.

Besides as a potential diagnostic tool for atherosclerosis, TSPO might also be a therapeutic target for atherosclerosis. The increased ROS and decreased antioxidants have been identified in high fat and high cholesterol atherogenic (HFHC) diet rat [77]. Interestingly, the oxidative stress caused by HFHC diet was accompanied by a reduction of TSPO-binding density [77]. This finding suggested that TSPO might also be a novel therapeutic target for atherosclerosis.

3.5. Myocarditis. Myocarditis is an inflammatory myocardial disease resulting from mainly viral infections and postviral immune-mediated responses [78-80]. TSPO is abundantly expressed in mast cells and macrophages [73-77]. In order to explore the effects of TSPO in immune response to myocarditis, a study has been conducted in BALB/c mice infected with coxsackievirus B3 (CVB3) [81]. TSPO is obviously decreased in infected male mice, and the elevated levels of PLA2, an endogenous TSPO ligand, can facilitate the activation of immune cells during the innate immune response to CVB3 infection [81]. Moreover, infected male mice have a greater expression of genes which are significant in regulating the influx of cholesterol into macrophages, such as PLA2 and the macrophage scavenger receptor [81]. Therefore, increased cholesterol metabolism associated with TSPO during the immune response to CVB3 infection may drive the proinflammatory reaction of myocarditis [81, 82]. However, the exact pathways, how TSPO ligands work, remain to be investigated.

3.6. Large Vessel Vasculitis. Large vessel vasculitis (LVV) is characterized by local chronic granulomatous inflammation of the vessel wall in aorta and its main branches [83-85]. TSPO is a potential diagnostic tool for LVV because of its high expression level in macrophages which are activated by cytokines $[85,86]$. Over the past two decades, ${ }^{11} \mathrm{C}-(\mathrm{R})-$ PK11195 or [N-methyl- $\left.{ }^{11} \mathrm{C}\right]-(\mathrm{R})-1$ - (2-chlorophenyl)-N- (1methylpropyl)-3-isoquinoline carboxamide, as a radioligand specifically binding to TSPO, has been extensively used to study neuroinflammation in a wide range of brain disorders $[87,88]$. Recently, a quantitative methodology of imaging LVV with ${ }^{11} \mathrm{C}$-(R)-PK11195 deriving from both plasma and image input functions has been developed [89, 90]. This approach demonstrated that active vasculitides in patients with systemic inflammatory disorders can be quantified by macrophage targeting ${ }^{11} \mathrm{C}-(\mathrm{R})-\mathrm{PK} 11195$. Moreover, the lack of significant uptake in asymptomatic patients confirmed the sensitivity and selectivity of this quantitative methodology [90]. However, further studies of reconstruction through partial-volume correction and respiration-gated acquisitions are highly needed to be conducted in a large population to minimize the vague influence of breathing on small lesions and to explore the diagnostic value of ${ }^{11} \mathrm{C}-(\mathrm{R})-\mathrm{PK} 11195$ in LVV.

\section{Conclusions}

TSPO regulates a wide range of biological functions in cardiovascular systems. Available TSPO ligands may be utilized as therapeutic drugs or diagnostic tools for CVD, including arrhythmias, MI, CH, atherosclerosis, myocarditis, and LVV [1-3].

The visible improvements of cardiac dysfunction have been achieved by TSPO ligands [1-3, 40, 42, 45]. The cardioprotective effects are related to ROS-release inhibition, mitochondrial energy provision, cardiac electrical activity retention, and intracellular calcium maintenance [1-5, 2528]. Although TSPO ligands could improve cardiac functions through preservation of mitochondrial physiologic effects which has been certified by a growing body of evidence, inconsistencies still exist, which might be due to different dosages and species used in studies from different groups [4, 33-39]. Therefore, future studies investigated in primate are highly needed prior to its clinical application.

In spite of the promising results from previous in vitro and in vivo studies, several limitations must be better addressed in the future. Firstly, what is the efficacy of middle/long-term use of TSPO ligands in CVD? Secondly, are there any potential adverse effects of TSPO ligands due to the definite high expression in other tissues, such as adrenal, kidney, and brain? Finally, do TSPO ligands really offer an improved benefit-risk profile compared with current treatments or diagnosis of CVD?

Nevertheless, given the vast range of potential applications, TSPO represents a novel therapeutic target and diagnostic tool of CVD in the near future.

\section{Authors' Contribution}

X. Qi and J. Xu and F. Wang contributed equally to this work. 


\section{Acknowledgments}

This work was supported by the grants from the National Natural Science Foundation of China (81200669 to J. Xiao; 81270314 to J. Xu), Innovation Program of Shanghai Municipal Education Commission (13YZ014 to J. Xiao) Foundation for University Young Teachers by Shanghai Municipal Education Commission (year 2012, to J. Xiao), Innovation Foundation of Shanghai University (A.10-0112-12-003 to J. Xiao) partially by Leading Academic Discipline Project of Shanghai Municipal Education Commission "Molecular Physiology."

\section{References}

[1] L. Veenman and M. Gavish, "The peripheral-type benzodiazepine receptor and the cardiovascular system. Implications for drug development," Pharmacology \& Therapeutics, vol. 110, no. 3, pp. 503-524, 2006.

[2] R. Rupprecht, V. Papadopoulos, G. Rammes et al., "Translocator protein $(18 \mathrm{kDa})$ (TSPO) as a therapeutic target for neurological and psychiatric disorders," Nature Reviews Drug Discovery, vol. 9, no. 12, pp. 971-988, 2010.

[3] V. Papadopoulos, M. Baraldi, T. R. Guilarte et al., "Translocator protein $(18 \mathrm{kDa})$ : new nomenclature for the peripheraltype benzodiazepine receptor based on its structure and molecular function," Trends in Pharmacological Sciences, vol. 27, no. 8, pp. 402-409, 2006.

[4] S. Surinkaew, S. Chattipakorn, and N. Chattipakorn, "Roles of mitochondrial benzodiazepine receptor in the heart," Canadian Journal of Cardiology, vol. 27, no. 2, pp. 262.e3262.e13, 2011.

[5] J. Fan, P. Lindemann, M. G. Feuilloley, and V. Papadopoulos, "Structural and functional evolution of the translocator protein (18 kDa)," Current Molecular Medicine, vol. 12, no. 4, pp. 369-386, 2012.

[6] A. Batarseh, J. Li, and V. Papadopoulos, "Protein kinase $\mathrm{C} \varepsilon$ regulation of translocator protein $(18 \mathrm{kDa})$ Tspo gene expression is mediated through a MAPK pathway targeting STAT3 and c-Jun transcription factors," Biochemistry, vol. 49, no. 23 , pp. 4766-4778, 2010.

[7] J. Li, J. Xiao, D. Liang et al., "Inhibition of mitochondrial translocator protein prevents atrial fibrillation," European Journal of Pharmacology, vol. 632, no. 1-3, pp. 60-64, 2010.

[8] F. G. Akar, M. A. Aon, G. F. Tomaselli, and B. O'Rourke, "The mitochondrial origin of postischemic arrhythmias," The Journal of Clinical Investigation, vol. 115, no. 12, pp. 35273535, 2005.

[9] D. A. Brown and B. O’Rourke, "Cardiac mitochondria and arrhythmias," Cardiovascular Research, vol. 88, no. 2, pp. 241249, 2010.

[10] M. A. Aon, S. Cortassa, F. G. Akar, and B. O’Rourke, "Mitochondrial criticality: a new concept at the turning point of life or death," Biochimica et Biophysica Acta, vol. 1762, no. 2, pp. 232-240, 2006.

[11] A. Jaiswal, S. Kumar, R. Enjamoori, S. Seth, A. K. Dinda, and S. K. Maulik, "Peripheral benzodiazepine receptor ligand Ro5-4864 inhibits isoprenaline-induced cardiac hypertrophy in rats," European Journal of Pharmacology, vol. 644, no. 1-3, pp. 146-153, 2010.

[12] O. Gaemperli, J. Shalhoub, D. R. Owen, F. Lamare, S. Johansson et al., "Imaging intraplaque inflammation in carotid atherosclerosis with ${ }^{11} \mathrm{C}$-PK11195 positron emission tomography/computed tomography," Europen Heart Journal, vol. 33, no. 15, pp. 1902-1910, 2012.

[13] I. Kindermann, C. Barth, F. Mahfoud, C. Ukena, M. Lenski et al., "Update on myocarditis," Journal of the American College of Cardiology, vol. 59, no. 9, pp. 779-792, 2012.

[14] S. Sagar, P. P. Liu, and L. T. Cooper Jr., "Myocarditis," The Lancet, vol. 379, no. 9817, pp. 738-747, 2012.

[15] F. Lamare, R. Hinz, O. Gaemperli et al., "Detection and quantification of large-vessel inflammation with ${ }^{11} \mathrm{C}-(\mathrm{R})$ PK11195 PET/CT," Journal of Nuclear Medicine, vol. 52, no. 1, pp. 33-39, 2011.

[16] J. Xu, J. Zhao, G. Evan, C. Xiao, Y. Cheng et al., "Circulating microRNAs: novel biomarkers for cardiovascular diseases," Journal of Molecular Medicine, vol. 90, no. 8, pp. 865-875, 2012.

[17] C. J. O’Donnell and E. G. Nabel, “Genomics of cardiovascular disease," The New England Journal of Medicine, vol. 365, pp. 2098-2109, 2011.

[18] J. L. Goldstein and M. S. Brown, "The LDL receptor," Arteriosclerosis, Thrombosis, and Vascular Biology, vol. 29, no. 4, pp. 431-438, 2009.

[19] C. P. Baines, R. A. Kaiser, T. Sheiko, W. J. Craigen, and J. D. Molkentin, "Voltage-dependent anion channels are dispensable for mitochondrial-dependent cell death," Nature Cell Biology, vol. 9, no. 5, pp. 550-555, 2007.

[20] J. E. Kokoszka, K. G. Waymire, S. E. Levy et al., "The ADP/ATP translocator is not essential for the mitochondrial permeability transition pore," Nature, vol. 427 , no. 6973 , pp. 461-465, 2004.

[21] O. R. Kunduzova, G. Escourrou, F. De La Farge et al., "Involvement of peripheral benzodiazepine receptor in the oxidative stress, death-signaling pathways, and renal injury induced by ischemia-reperfusion," Journal of the American Society of Nephrology, vol. 15, no. 8, pp. 2152-2160, 2004.

[22] E. G. Jordà, A. Jiménez, E. Verdaguer et al., "Evidence in favour of a role for peripheral-type benzodiazepine receptor ligands in amplification of neuronal apoptosis," Apoptosis, vol. 10, no. 1, pp. 91-104, 2005.

[23] L. Betti, G. Giannaccini, M. Nigro, S. Dianda, V. Gremigni, and A. Lucacchini, "Studies of peripheral benzodiazepine receptors in mussels: comparison between a polluted and a nonpolluted site," Ecotoxicology and Environmental Safety, vol. 54, no. 1, pp. 36-42, 2003.

[24] S. Galiègue, P. Casellas, A. Kramar, N. Tinel, and J. SimonyLafontaine, "Immunohistochemical assessment of the peripheral benzodiazepine receptor in breast cancer and its relationship with survival," Clinical Cancer Research, vol. 10, no. 6, pp. 2058-2064, 2004.

[25] L. Veenman, E. Levin, G. Weisinger et al., "Peripheral-type benzodiazepine receptor density and in vitro tumorigenicity of glioma cell lines," Biochemical Pharmacology, vol. 68, no. 4, pp. 689-698, 2004.

[26] N. Leducq, F. Bono, T. Sulpice et al., "Role of peripheral benzodiazepine receptors in mitochondrial, cellular, and cardiac damage induced by oxidative stress and ischemiareperfusion," Journal of Pharmacology and Experimental Therapeutics, vol. 306, no. 3, pp. 828-837, 2003.

[27] D. Kletsas, W. Li, Z. Han, and V. Papadopoulos, "Peripheraltype benzodiazepine receptor (PBR) and PBR drug ligands in fibroblast and fibrosarcoma cell proliferation: role of ERK, c-Jun and ligand-activated PBR-independent pathways," Biochemical Pharmacology, vol. 67, no. 10, pp. 1927-1932, 2004. 
[28] M. K. Chen and T. R. Guilarte, "Translocator protein $18 \mathrm{kDa}$ (TSPO): molecular sensor of brain injury and repair," Pharmacology \& Therapeutics, vol. 118, no. 1, pp. 1-17, 2008.

[29] K. Saegusa, Y. Furukawa, and Y. Ogiwara, "Pharmacologic basis of responses to midazolam in the isolated, crossperfused, canine right atrium," Anesthesia and Analgesia, vol. 66, no. 8, pp. 711-718, 1987.

[30] Y. Edoute, J. Giris, S. A. Ben-Haim et al., "Ro 5-4864 and PK 11195, but not diazepam, depress cardiac function in an isolated working rat heart model," Pharmacology, vol. 46, no. 4, pp. 224-230, 1993.

[31] M. Mestre, T. Carriot, C. Belin et al., "Electrophysiological and pharmacological evidence that peripheral type benzodiazepine receptors are coupled to calcium channels in the heart," Life Sciences, vol. 36, no. 4, pp. 391-400, 1985.

[32] J. Li, J. Xiao, Y. Liu et al., "Mitochondrial benzodiazepine receptors mediate cardioprotection of estrogen against ischemic ventricular fibrillation," Pharmacological Research, vol. 60, no. 1, pp. 61-67, 2009.

[33] I. L. Grupp, J. F. French, and M. A. Matlib, "Benzodiazepine Ro 5-4864 increases coronary flow," European Journal of Pharmacology, vol. 143, no. 1, pp. 143-147, 1987.

[34] B. A. Weissman, G. T. Bolger, and P. K. Chiang, "Interactions between nitrogen oxide-containing compounds and peripheral benzodiazepine receptors," FEBS Letters, vol. 260, no. 2, pp. 169-172, 1990.

[35] M. Mestre, T. Carriot, C. Belin et al., "Electrophysiological and pharmacological characterization of peripheral benzodiazepine receptors in a guinea pig heart preparation," Life Sciences, vol. 35, no. 9, pp. 953-962, 1984.

[36] M. Holck and W. Osterrieder, "The peripheral, high affinity benzodiazepine binding site is not coupled to the cardiac $\mathrm{Ca}^{2+}$ channel," European Journal of Pharmacology, vol. 118, no. 3, pp. 293-301, 1985.

[37] D. A. Brown, M. A. Aon, F. G. Akar, T. Liu, N. Sorarrain, and B. O'Rourke, "Effects of 4 '-chlorodiazepam on cellular excitation-contraction coupling and ischaemia-reperfusion injury in rabbit heart," Cardiovascular Research, vol. 79, no. 1, pp. 141-149, 2008.

[38] R. S. Leeuwin, A. Zeegers, J. van Hamme, and H. van Wilgenburg, "Modification of cardiac actions of RO 05-4864 by PK 11195 and flumazenil in the perfused rat heart," Life Sciences, vol. 61, no. 17, pp. 1631-1642, 1997.

[39] P. Charbonneau, A. Syrota, C. Crouzel et al., "Peripheral-type benzodiazepine receptors in the living heart characterized by positron emission tomography," Circulation, vol. 73, no. 3, pp. 476-483, 1986.

[40] M. L. James, S. Selleri, and M. Kassiou, "Development of ligands for the peripheral benzodiazepine receptor," Current Medicinal Chemistry, vol. 13, no. 17, pp. 1991-2001, 2006.

[41] R. B. Banati, "Visualising microglial activation in vivo," Glia, vol. 40, no. 2, pp. 206-217, 2002.

[42] S. Schaller, S. Paradis, G. A. Ngoh et al., "TRO40303, a new cardioprotective compound, inhibits mitochondrial permeability transition," Journal of Pharmacology and Experimental Therapeutics, vol. 333, no. 3, pp. 696-706, 2010.

[43] M. A. Aon, S. Cortassa, F. G. Akar, D. A. Brown, L. Zhou, and B. O’Rourke, "From mitochondrial dynamics to arrhythmias," The International Journal of Biochemistry \& Cell Biology, vol. 41, no. 10, pp. 1940-1948, 2009.

[44] C. Schmidt, J. Kisselbach, P. A. Schweizer, H. A. Katus, and D. Thomas, "The pathology and treatment of cardiac arrhythmias: focus on atrial fibrillation," Vascular Health and Risk Management, vol. 7, pp. 193-202, 2011.
[45] L. S. Wann, A. B. Curtis, C. T. January et al., "2011 ACCF/AHA/HRS focused update on the management of patients with atrial fibrillation (updating the 2006 guideline): a report of the American college of cardiology foundation/American heart association task force on practice guidelines," Circulation, vol. 123, no. 1, pp. 104-123, 2011.

[46] J. Li, J. Xu, J. Xiao et al., "Preservation of TSPO by chronic intermittent hypobaric hypoxia confers antiarrhythmic activity," Journal of Cellular and Molecular Medicine, vol. 15, no. 1, pp. 134-140, 2011.

[47] N. R. Brady, A. Hamacher-Brady, H. V. Westerhoff, and R. A. Gottlieb, "A wave of reactive oxygen species (ROS)-induced ROS release in a sea of excitable mitochondria," Antioxidants and Redox Signaling, vol. 8, no. 9-10, pp. 1651-1665, 2006.

[48] D. B. Zorov, M. Juhaszova, and S. J. Sollott, "Mitochondrial ROS-induced ROS release: an update and review," Biochimica et Biophysica Acta, vol. 1757, no. 5-6, pp. 509-517, 2006.

[49] J. Cho, K. Won, D. Wu et al., "Potent mitochondria-targeted peptides reduce myocardial infarction in rats," Coronary Artery Disease, vol. 18, no. 3, pp. 215-220, 2007.

[50] M. A. Aon, S. Cortassa, C. Maack, and B. O’Rourke, “Sequential opening of mitochondrial ion channels as a function of glutathione redox thiol status," The Journal of Biological Chemistry, vol. 282, no. 30, pp. 21889-21900, 2007.

[51] M. A. Aon, S. Cortassa, E. Marbán, and B. O’Rourke, “Synchronized whole cell oscillations in mitochondrial metabolism triggered by a local release of reactive oxygen species in cardiac myocytes," The Journal of Biological Chemistry, vol. 278, no. 45, pp. 44735-44744, 2003.

[52] M. A. Aon, S. Cortassa, and B. O’Rourke, "Mitochondrial oscillations in physiology and pathophysiology," Advances in Experimental Medicine and Biology, vol. 641, pp. 98-117, 2008.

[53] D. A. Brown, M. A. Aon, C. R. Frasier et al., "Cardiac arrhythmias induced by glutathione oxidation can be inhibited by preventing mitochondrial depolarization," Journal of Molecular and Cellular Cardiology, vol. 48, no. 4, pp. 673-679, 2010.

[54] C. Piot, P. Croisille, P. Staat et al., "Effect of cyclosporine on reperfusion injury in acute myocardial infarction," The New England Journal of Medicine, vol. 359, no. 5, pp. 473-481, 2008.

[55] G. E. Billman, "The cardiac sarcolemmal ATP-sensitive potassium channel as a novel target for anti-arrhythmic therapy," Pharmacology \& Therapeutics, vol. 120, no. 1, pp. 54-70, 2008.

[56] E. Dongó, I. Hornyák, Z. Benko, and L. Kiss, "The cardioprotective potential of hydrogen sulfide in myocardial ischemia/reperfusion injury," Acta Physiologica Hungarica, vol. 98, no. 4, pp. 369-381, 2011.

[57] D. A. Liem, H. M. Honda, J. Zhang, D. Woo, and P. Ping, "Past and present course of cardioprotection against ischemiareperfusion injury," Journal of Applied Physiology, vol. 103, no. 6, pp. 2129-2136, 2007.

[58] P. Ferdinandy, R. Schulz, and G. F. Baxter, "Interaction of cardiovascular risk factors with myocardial ischemia/reperfusion injury, preconditioning, and postconditioning," Pharmacological Reviews, vol. 59, no. 4, pp. 418-458, 2007.

[59] A. P. Halestrap, "What is the mitochondrial permeability transition pore?" Journal of Molecular and Cellular Cardiology, vol. 46, no. 6, pp. 821-831, 2009.

[60] F. Ricchelli, J. Šileikyte, and P. Bernardi, "Shedding light on the mitochondrial permeability transition," Biochimica et Biophysica Acta, vol. 1807, no. 5, pp. 482-490, 2011. 
[61] L. Argaud, O. Gateau-Roesch, D. Muntean et al., "Specific inhibition of the mitochondrial permeability transition prevents lethal reperfusion injury," Journal of Molecular and Cellular Cardiology, vol. 38, no. 2, pp. 367-374, 2005.

[62] N. Oka, L. Wang, W. Mi, W. Zhu, O. Honjo, and C. A. Caldarone, "Cyclosporine A prevents apoptosis-related mitochondrial dysfunction after neonatal cardioplegic arrest," The Journal of Thoracic and Cardiovascular Surgery, vol. 135, no. 1, pp. 123.e2-130.e2, 2008.

[63] J. Šileikyte, V. Petronilli, A. Zulian et al., "Regulation of the inner membrane mitochondrial permeability transition by the outer membrane translocator protein (peripheral benzodiazepine receptor)," The Journal of Biological Chemistry, vol. 286, no. 2, pp. 1046-1053, 2011.

[64] J. Xiao, D. Liang, H. Zhang, Y. Liu, F. Li, and Y. H. Chen, " 4 ' chlorodiazepam, a translocator protein $(18 \mathrm{kDa})$ antagonist, improves cardiac functional recovery during postischemia reperfusion in rats," Experimental Biology and Medicine, vol. 235, no. 4, pp. 478-486, 2010.

[65] F. N. Obame, R. Zini, R. Souktani, A. Berdeaux, and D. Morin, "Peripheral benzodiazepine receptor-induced myocardial protection is mediated by inhibition of mitochondrial membrane permeabilization," Journal of Pharmacology and Experimental Therapeutics, vol. 323, no. 1, pp. 336-345, 2007.

[66] S. K. Maulik and S. Kumar, "Oxidative stress and cardiac hypertrophy: a review," Toxicology Mechanisms Methods, vol. 22, pp. 359-366, 2012.

[67] J. F. X. Ainscough, M. J. Drinkhill, A. Sedo et al., "Angiotensin II type-1 receptor activation in the adult heart causes blood pressure-independent hypertrophy and cardiac dysfunction," Cardiovascular Research, vol. 81, no. 3, pp. 592-600, 2009.

[68] M. P. Gupta, "Factors controlling cardiac myosin-isoform shift during hypertrophy and heart failure," Journal of Molecular and Cellular Cardiology, vol. 43, no. 4, pp. 388-403, 2007.

[69] H. Tsutsui, S. Kinugawa, and S. Matsushima, "Mitochondrial oxidative stress and dysfunction in myocardial remodelling," Cardiovascular Research, vol. 81, no. 3, pp. 449-456, 2009.

[70] D. Badenhorst, D. Veliotes, M. Maseko et al., “ $\beta$-adrenergic activation initiates chamber dilatation in concentric hypertrophy," Hypertension, vol. 41, no. 3, pp. 499-504, 2003.

[71] E. A. van Vré, I. van Brussel, J. M. Bosmans et al., "Dendritic cells in human atherosclerosis: from circulation to atherosclerotic plaques," Mediators of Inflammation, vol. 2011, Article ID 941396, 13 pages, 2011.

[72] E. Thorp, M. Subramanian, and I. Tabas, "The role of macrophages and dendritic cells in the clearance of apoptotic cells in advanced atherosclerosis," European Journal of Immunology, vol. 41, no. 9, pp. 2515-2518, 2011.

[73] Y. Fujimura, P. M. Hwang, H. Trout III et al., "Increased peripheral benzodiazepine receptors in arterial plaque of patients with atherosclerosis: an autoradiographic study with $\left[{ }^{3} \mathrm{H}\right]$ PK 11195," Atherosclerosis, vol. 201, no. 1, pp. 108-111, 2008.

[74] J. G. Park and G. T. Oh, "The role of peroxidases in the pathogenesis of atherosclerosis," BMB Reports, vol. 44, no. 8, pp. 497-505, 2011.

[75] J. L. E. Bird, D. Izquierdo-Garcia, J. R. Davies et al., "Evaluation of translocator protein quantification as a tool for characterising macrophage burden in human carotid atherosclerosis," Atherosclerosis, vol. 210, no. 2, pp. 388-391, 2010.
[76] I. Laitinen, P. Marjamäki, K. Någren et al., "Uptake of inflammatory cell marker $\left[{ }^{11} \mathrm{C}\right] \mathrm{PK} 11195$ into mouse atherosclerotic plaques," European Journal of Nuclear Medicine and Molecular Imaging, vol. 36, no. 1, pp. 73-80, 2009.

[77] J. Dimitrova-Shumkovska, L. Veenman, T. Ristoski, S. Leschiner, and M. Gavish, "Chronic high fat, high cholesterol supplementation decreases $18 \mathrm{kDa}$ translocator protein binding capacity in association with increased oxidative stress in rat liver and aorta," Food and Chemical Toxicology, vol. 48, no. 3, pp. 910-921, 2010.

[78] D. Fatkin and members of the CSANZ Cardiac Genetic Diseases Council Writing Group, "Guidelines for the diagnosis and management of familial dilated cardiomyopathy," Heart, Lung and Circulation, vol. 20, no. 11, pp. 691-693, 2011.

[79] M. Movsesian, O. Wever-Pinzon, and F. Vandeput, "PDE3 inhibition in dilated cardiomyopathy," Current Opinion in Pharmacology, vol. 11, no. 6, pp. 707-713, 2011.

[80] A. Fabre and M. N. Sheppard, "Sudden adult death syndrome and other non-ischaemic causes of sudden cardiac death," Heart, vol. 92, no. 3, pp. 316-320, 2006.

[81] J. A. Onyimba, M. J. Coronado, A. E. Garton, J. B. Kim, A. Bucek et al., "The innate immune response to coxsackievirus B3 predicts progression to cardiovascular disease and heart failure in male mice," Biology of Sex Differences, vol. 2, article 2, 2011.

[82] S. Frisancho-Kiss, S. E. Davis, J. F. Nyland et al., "Cutting edge: cross-regulation by TLR4 and T cell Ig mucin-3 determines sex differences in inflammatory heart disease," The Journal of Immunology, vol. 178, no. 11, pp. 6710-6714, 2007.

[83] N. Pipitone, A. Versari, and C. Salvarani, "Role of imaging studies in the diagnosis and follow-up of large-vessel vasculitis: an update," Rheumatology, vol. 47, no. 4, pp. 403-408, 2008.

[84] A. Gulati and A. Bagga, "Large vessel vasculitis," Pediatric Nephrology, vol. 25, no. 6, pp. 1037-1048, 2010.

[85] L. Caspary and S. Schellong, "Large vessel vasculitis," Der Internist, vol. 50, no. 8, pp. 958-963, 2009.

[86] K. Sano, “Takayasu disease-pulseless disease," Brain and Nerve, vol. 60, no. 1, pp. 31-35, 2008.

[87] A. Gerhard, J. Schwarz, R. Myers, R. Wise, and R. B. Banati, "Evolution of microglial activation in patients after ischemic stroke: a $\left[{ }^{11} \mathrm{C}\right](\mathrm{R})-\mathrm{PK} 11195$ PET study," NeuroImage, vol. 24, no. 2, pp. 591-595, 2005.

[88] B. N. van Berckel, M. G. Bossong, R. Boellaard et al., "Microglia activation in recent-onset schizophrenia: a quantitative (R)-[ $\left.{ }^{11} \mathrm{C}\right] \mathrm{PK} 11195$ positron emission tomography study," Biological Psychiatry, vol. 64, no. 9, pp. 820-822, 2008.

[89] F. Pugliese, O. Gaemperli, A. R. Kinderlerer et al., "Imaging of vascular inflammation with $\left[{ }^{11} \mathrm{C}\right]-\mathrm{PK} 11195$ and positron emission tomography/computed tomography angiography," Journal of the American College of Cardiology, vol. 56, no. 8, pp. 653-661, 2010.

[90] Y. Fujimura, P. M. Hwang, H. Trout III et al., "Increased peripheral benzodiazepine receptors in arterial plaque of patients with atherosclerosis: an autoradiographic study with $\left[{ }^{3} \mathrm{H}\right] \mathrm{PK}$ 11195," Atherosclerosis, vol. 201, no. 1, pp. 108-111, 2008. 


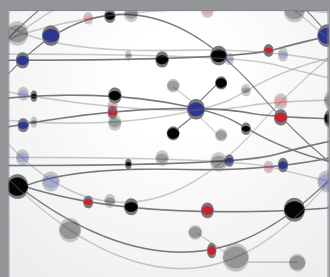

The Scientific World Journal
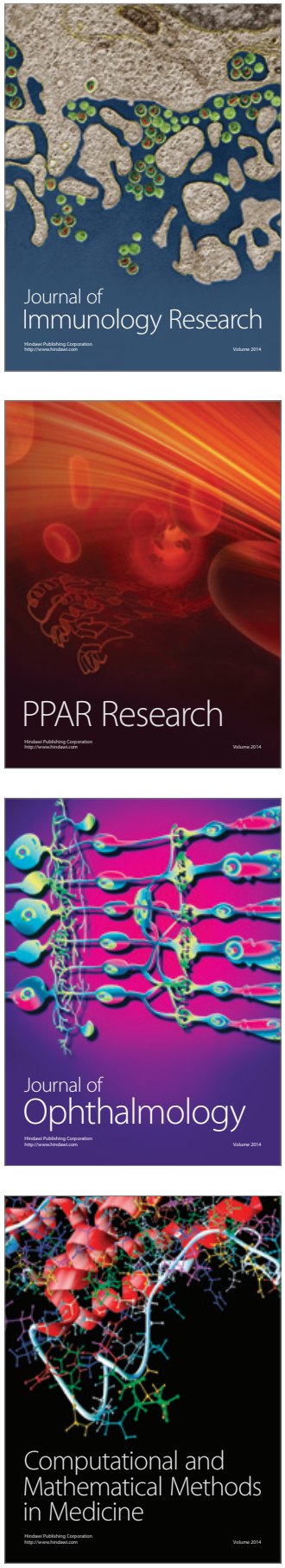

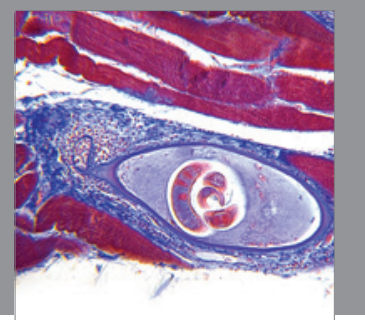

Gastroenterology

Research and Practice
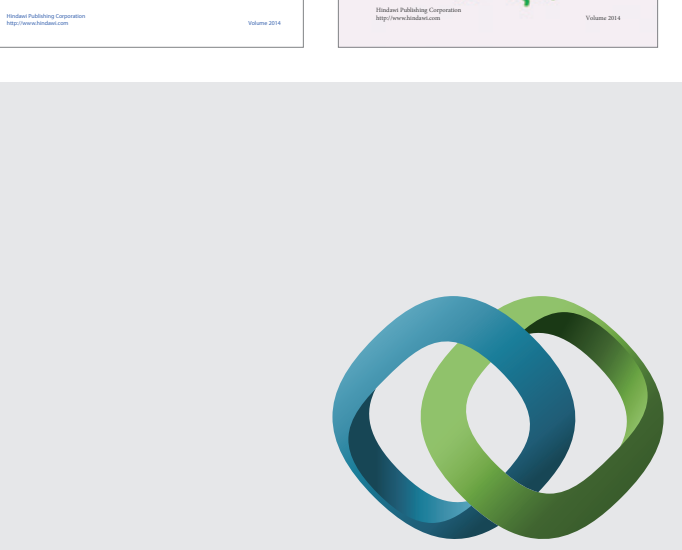

\section{Hindawi}

Submit your manuscripts at

http://www.hindawi.com
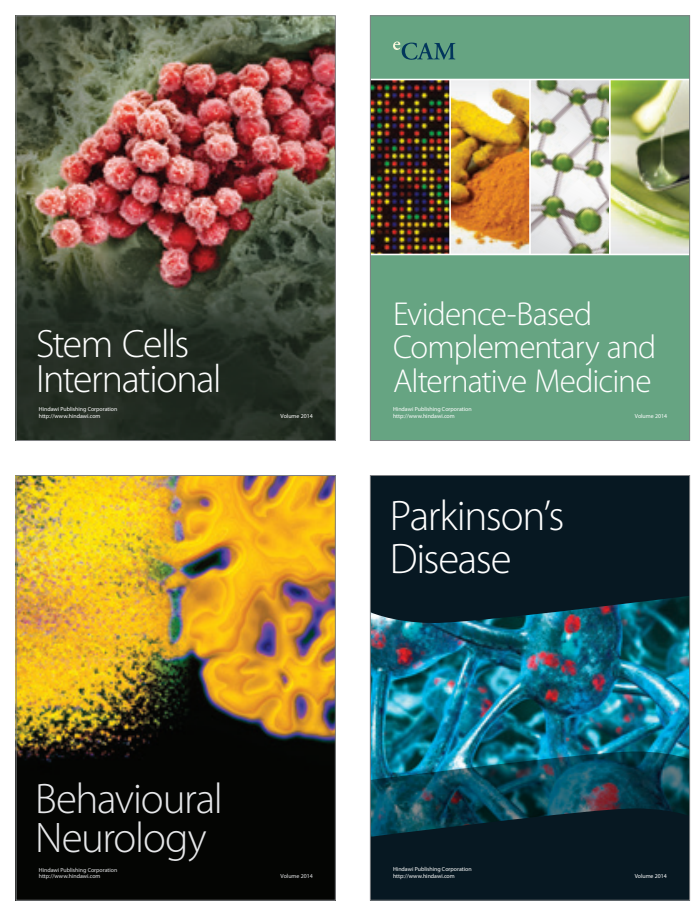

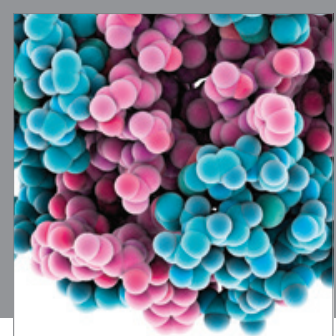

Journal of
Diabetes Research

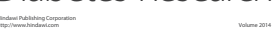

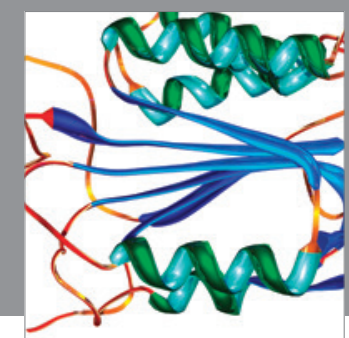

Disease Markers
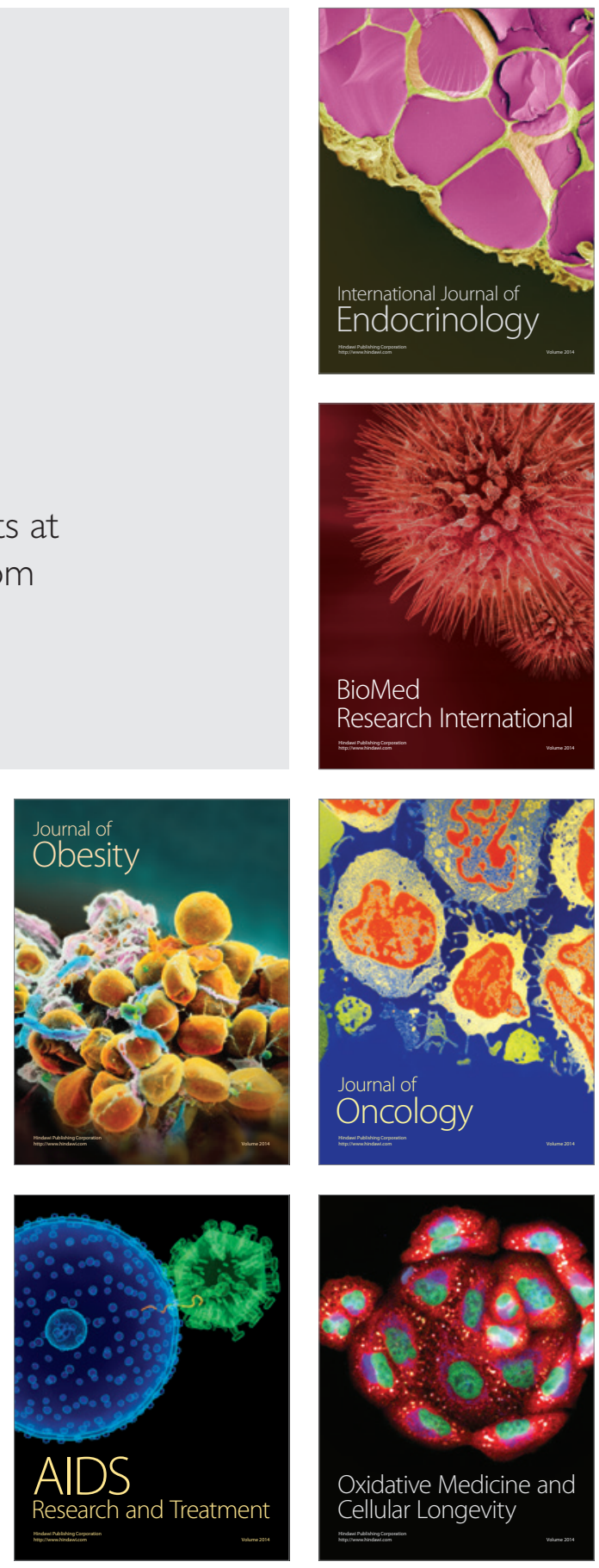